\title{
Capacity of Data Collection in Wireless Sensor Networks Based on Mutual Information and MMSE Estimation
}

\author{
Ajib Setyo Arifin and Tomoaki Ohtsuki \\ Graduate School of Science and Technology, Keio University, 3-14-1 Hiyoshi, Kohoku-ku, Yokohama 223-8522, Japan \\ Correspondence should be addressed to Tomoaki Ohtsuki; ohtsuki@ics.keio.ac.jp
}

Received 4 December 2013; Accepted 30 December 2013; Published 17 February 2014

Academic Editors: T.-S. Chen, J. Li, and Y. Yu

Copyright (C) 2014 A. S. Arifin and T. Ohtsuki. This is an open access article distributed under the Creative Commons Attribution License, which permits unrestricted use, distribution, and reproduction in any medium, provided the original work is properly cited.

\begin{abstract}
We investigate the properties of data collection in wireless sensor networks, in terms of both capacity and power allocation strategy. We consider a scenario in which a number of sensors observe a target being estimated at fusion center (FC) using minimum meansquare error (MMSE) estimator. Based on the relationship between mutual information and MMSE (I-MMSE), the capacity of data collection in coherent and orthogonal multiple access channel (MAC) models is derived. Considering power constraint, the capacity is derived under two scenarios: equal power allocation and optimal power allocation of both models. We provide the upper bound of capacity as a benchmark. In particular, we show that the capacity of data collection scales as $\Theta((1 / 2) \log (1+L))$ when the number of sensors $L$ grows to infinity. We show through simulation results that for both coherent and orthogonal MAC models, the capacity of the optimal power is larger than that of the equal power. We also show that the capacity of coherent MAC is larger than that of orthogonal MAC, particularly when the number of sensors $L$ is large and the total power $P$ is fixed.
\end{abstract}

\section{Introduction}

Wireless sensor networks (WSNs) consisting of a large number of nodes are usually deployed in a large region for many applications, such as surveillance, security, and environmental monitoring. The goal of a sensor network is often to deliver the sensing data from all sensors to a fusion center (FC) and then conduct further analysis at the FC. Thus, data collection is important in sensor network applications [1]. Theoretical measure that captures the limits of collection processing in sensor network is the capacity of data collection. Capacity of data collection reflects how fast FC can collect sensing data from all sensors [2]. Understanding the capacity of the network is important for network designers in a feasibility of a large scale network deployment [3], particularly, to improve the performance of WSNs [1]. Furthermore, such understanding is essential in the development of efficient protocols [4].

Capacity limits of data collection in wireless sensor networks have been studied in the literature [1-10]. In [4, 5], they introduced the transport capacity of many-to-one in dense sensor networks. The authors in $[6,7]$ investigated the capacity of data collection with complex physical layer techniques. The capacity that involves multiple selected sources and destination has been studied in [8]. The capacity of data collection of single and multisinks (FC) is investigated [9]. In [2], the authors derive capacity of data collection in arbitrary WSNs. A data collection capacity that considers delay and compressive sensing has been, recently, investigated in [10]. Most of the literature resources calculate capacity based on either the physical models or the protocol model. Physical model also known as the signal-to-interference-plus noise ratio (SINR) model, is based on practical tranceiver designs of communication system that treats interference as noise. Further, capacity calculation is based on Shannon's formula. The other model is the protocol model. The model states that a successful transmission occurs when a sensor falls inside the transmission range of its intended transmitter and falls outside the interference ranges of other nonintended transmitters. However, the protocol model is relatively inaccurate, when simultaneous transmissions are allowed in the network $[3,11]$.

Study of distributed estimation in WSNs is one of the interesting topics that many researchers are working on. Some of the results are listed in [12-23]. Some literature 
addresses digital sensor transmission, where the noisecorrupted sensor observations are quantized into bits and digitally transmitted to the FC [12-18]. In [19-23], they consider analog sensor transmission, where the sensors amplify and forward the observations to the FC, and the performance of estimation is generally better than that of the digital transmission. Distributed estimation by considering MAC model has been considered in $[24,25]$. They reveal that distributed estimation using the coherent MAC is more bandwidth efficient than the orthogonal MAC.

Another important property of many WSNs is their stringent power constraint. In such networks, sensors have only small-size batteries whose replacement can be costly. Thus, sensor network operations must be energy efficient to maximize network lifetime. However, there are only a few authors that consider power constraint in deriving the capacity of data collection. In [6], they characterized the transport capacity of many-to-one dense wireless networks subject to a constraint on the total power. The energy efficiency and data latency are considered in [3] for designing data gathering capacity. However, they still do not provide how to allocate the power optimally.

In this paper, we focus on deriving capacity of data collection for random networks under coherent and orthogonal MAC scenario based on equality of mutual informationminimum mean-squared error estimation (I-MMSE). We provide a new perspective of capacity calculation of data collection in WSNs that can be derived from error estimation of the target at the FC. The relationship between mutual information and MMSE has been revealed by Guo et al. in [26]. First, we derive a capacity formulation on coherent MAC model. In coherent MAC model, we assume that there is perfect synchronization between sensors and the fusion center so that the transmitted messages from local sensors can be coherently combined at the fusion center. With such an assumption, one key design consideration at local sensors and the fusion center is how to jointly process the sensed and received information in terms of capacity. We write a problem formulation for maximizing the capacity and then solve it through convex optimization technique. We derive the optimal power allocation strategy to maximize the capacity. The upper bound on the capacity of data collection with coherent MAC model is also derived as a benchmark. Second, we derive a capacity formulation on orthogonal MAC scenario. The motivation for using orthogonal multiple access schemes such as Frequency Division Multiple Access (FDMA) is the removal of the requirement on the carrier level synchronization among sensors [25]. As the coherent MAC model, we also derive optimal power allocation strategy for the case where the capacity is maximized under certain power constraints. In the orthogonal model, the optimal power allocation is achieved by turning off certain sensors with bad channels and bad observation quality. The upper bound on this model is also derived and interestingly equal to the upper bound on coherent one.

The rest of the paper is organized as follows. Section 2 describes the preliminary theory and system model. In Section 3, we formulate the capacity of data collection for the upper bound, equal power allocation, and optimal power allocation in coherent MAC model. In Section 4 we formulate the capacity of data collection for the equal power allocation, optimal power allocation, and the upper bound on orthogonal MAC model. Section 5 presents some simulation results and conclusion is drawn in Section 6.

\section{Problem Formulation}

As a preliminary, we start by explaining the relationship between mutual information and MMSE [26].

2.1. Capacity of the Gaussian Channel Based I-MMSE Approach. An input-output model can be written as

$$
Y=\sqrt{\operatorname{snr}} X+N
$$

where $N \sim \mathcal{N}(0,1)$ is standard Gaussian. We note here that snr in (1) coincides with the usual notation of signal-to-noise power ratio (SNR) only if $E\left[X^{2}\right]=1$. Then, we refer to snr as SNR regardless of the input power. The MMSE of estimating the input $X$ of the model given the noisy output $Y$ can be denoted by

$$
\begin{aligned}
\operatorname{mmse}(X, \mathrm{snr}) & =\operatorname{mmse}(X \mid \sqrt{\mathrm{snr}}+N) \\
& =E\left[(X-E[X \mid \sqrt{\mathrm{snr}}+N])^{2}\right],
\end{aligned}
$$

where $E[\cdot]$ is the expected value.

The MMSE can be regarded as a function of SNR for every given distribution $P_{X}$. Particularly, if $X \sim \mathcal{N}\left(m, \sigma_{X}^{2}\right)$, the MMSE can be denoted by

$$
\operatorname{mmse}(X, \mathrm{snr})=\frac{\sigma_{X}^{2}}{1+\sigma_{X}^{2} \mathrm{snr}} .
$$

Moreover, simple quantitative connections between MMSE and information measures are revealed in [26]. One of the results is

$$
2 \frac{d}{d \operatorname{snr}} I(X ; Y)=\operatorname{mmse}(X, \mathrm{snr})
$$

for every $s n r \geq 0$. The corresponding capacity of the model is

$$
\begin{aligned}
C & =\max I(X ; Y) \\
& =\frac{1}{2} \int_{0}^{\mathrm{snr}} \operatorname{mmse}(X, \mathrm{snr}) d \mathrm{snr} \\
& =\frac{1}{2} \log \left(1+\sigma_{X}^{2} \mathrm{snr}\right)
\end{aligned}
$$

where we adopt natural logarithms and use nats as the unit of all capacity measures.

2.2. System Model. Suppose that there are $L$ sensors, each making observation on a common unknown parameter $s$ as in Figure 1. The sensors observe $s$ with noisy observation $n$ that has zero mean and variance, $\sigma_{n_{i}}^{2}$. We assume the sensor and FC communicate with coherent MAC. When source and 


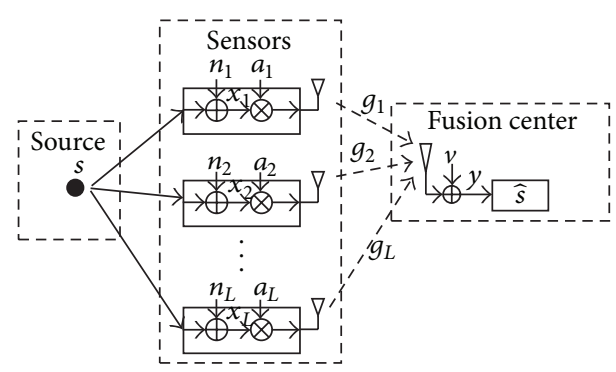

FIGURE 1: System model of the capacity of data collection in WSNs.

observation are scalars, the observation model can be written as

$$
x_{i}=s+n_{i}, \quad 1 \leq i \leq L .
$$

Suppose that the corresponding analog amplifying and forwarding scheme is used; we have a power amplification factor $a_{i}$ of $i$ th sensor. The average transmit power of sensor $i$ is

$$
\begin{aligned}
P_{i} & =a_{i}^{2}\left(\sigma_{s}^{2}+\sigma_{n_{i}}^{2}\right) \\
& =a_{i}^{2}\left(1+\alpha_{i}^{-1}\right),
\end{aligned}
$$

where we assume that $\sigma_{s}^{2}=1 . \alpha_{i}=1 / \sigma_{n_{i}}^{2}$ is SNR observation of sensor $i$. After amplification, signals are transmitted to the FC. The received signal at FC is

$$
\begin{aligned}
y & =\sum_{i=1}^{L} g_{i} a_{i} x_{i}+v \\
& =\sum_{i=1}^{L} g_{i} a_{i} s+\sum_{i=1}^{L} g_{i} a_{i} n_{i}+v,
\end{aligned}
$$

where $g_{i}$ and $v$ are channel gain and channel noise, respectively. Similarly, $v$ is assumed to have zero mean and unit variance, $\sigma_{v}^{2}$. The linear MMSE estimator of $s$ from $y$ is $\widehat{s}=$ $\left(E[s y] / E\left[y^{2}\right]\right) y$, which has MSE, $J$, satisfying [24]

$$
\frac{1}{J}=1+\left(1+\sum_{i=1}^{L} g_{i}^{2} a_{i}^{2}\right)^{-1}\left(\sum_{i=1}^{L} g_{i} a_{i} \sqrt{\alpha_{i}}\right)^{2} .
$$

\section{Capacity of Data Collection in Coherent MAC Model}

3.1. Upper Bound on Capacity of Data Collection. Note that when all sensor observations $x_{i}=\left[x_{1}, \ldots, x_{L}\right]^{T}$ are directly available to the FC, a centralized estimator $\widehat{s}_{0}=$ $\left(E[s x] / E\left[x^{2}\right]\right) x$, achieves an MSE, $J_{0}$, as

$$
\begin{aligned}
\frac{1}{J_{0}} & =1+\sum_{i=1}^{L} \alpha_{i} \\
& =1+\gamma,
\end{aligned}
$$

where $\alpha_{i}$ and $\gamma$ are SNR observation of sensor $i$ and the total SNR observation, respectively. Analytical proof is also available in Appendix A. Applying (5), we can express the capacity of data collection as follows:

$$
\begin{aligned}
C_{\text {upp } 1} & =\frac{1}{2} \int_{0}^{\mathrm{SNR}_{\mathrm{tot}}} J_{0} d \gamma \\
& =\frac{1}{2} \int_{0}^{\mathrm{SNR}} \frac{1}{1+\gamma} d \gamma \\
& =\frac{1}{2} \log \left(1+\mathrm{SNR}_{\mathrm{tot}}\right) .
\end{aligned}
$$

Because of the randomness of sensors deployment, we assume that noisy observation becomes i.i.d., with $\sigma_{n_{1}}^{2}, \ldots, \sigma_{n_{L}}^{2}=$ $\sigma_{n}^{2}$. Then, the upper bound of the capacity of data collection can be expressed as

$$
C_{\text {upp1 }}=\frac{1}{2} \log \left(1+\frac{L}{\sigma_{n}^{2}}\right) .
$$

Without loss of generality, we can express the capacity of the network scaled by $\Theta((1 / 2) \log (1+L))$ as the number of sensors becomes infinity, $L \rightarrow \infty$.

3.2. Capacity of Data Collection for Equal Power Allocation. Suppose all sensors use the same transmit power, $P_{i}=P / L$, where $P$ is the total transmit power. From (7), we get $a_{i}=$ $\sqrt{P / L\left(\alpha_{i}^{-1}+1\right)}$. Let $J_{u}(P)$ denote the achieved MSE with equal transmit power. From $(9), J_{\text {eql }}(P)$ satisfies

$$
\begin{aligned}
\frac{1}{J_{\text {eq } 1}(P)}= & +\left(\frac{L}{P}+\sum_{i=1}^{L} \frac{g_{i}^{2}}{\alpha_{i}^{-1}+1}\right)^{-1} \\
& \times\left(\sum_{i=1}^{L} \sqrt{\frac{\alpha_{i}}{\alpha_{i}^{-1}+1}} g_{i}\right)^{2} .
\end{aligned}
$$

With the same analogy in (10), we define $\left(L / P+\sum_{i=1}^{L}\left(g_{i}^{2} /\left(\alpha_{i}^{-1}+1\right)\right)\right)^{-1}\left(\sum_{i=1}^{L} \sqrt{\left(\alpha_{i} /\left(\alpha_{i}^{-1}+1\right)\right)} g_{i}\right)^{2}$ as a total SNR of the system. Therefore, we can express the capacity of equal transmit power as follows:

$$
\begin{aligned}
C_{\mathrm{eq} 1}(P)=\frac{1}{2} \log ( & +\left(\frac{L}{P}+\sum_{i=1}^{L} \frac{g_{i}^{2}}{\alpha_{i}^{-1}+1}\right)^{-1} \\
& \times\left(\sum_{i=1}^{L} \sqrt{\frac{\alpha_{i}}{\alpha_{i}^{-1}+1}} g_{i}\right)^{2} .
\end{aligned}
$$

For $P \rightarrow \infty$, we can write the capacity as

$$
\begin{aligned}
C_{\text {eq } 1}(\infty)= & \frac{1}{2} \log \left(1+\left(\sum_{i=1}^{L} \frac{g_{i}^{2}}{\alpha_{i}^{-1}+1}\right)^{-1}\right. \\
& \left.\times\left(\sum_{i=1}^{L} \sqrt{\frac{\alpha_{i}}{\alpha_{i}^{-1}+1}} g_{i}\right)^{2}\right) \\
\leq &
\end{aligned}
$$


We can summarize the results on (12), (14), and (15) where each sensor uses exactly the same transmit power of $P / L$. We can express for every finite $P$ as

$$
C_{\text {upp } 1} \geq C_{\text {eq } 1}(\infty) \geq C_{\text {eq1 }}(P) .
$$

3.3. Capacity of Data Collection for Optimal Power Allocation. Here, we consider an optimal power allocation whereby the transmit power is optimally allocated among the sensors to achieve the maximum capacity. From the right hand side (RHS) of (9), we denote by $\beta=$ $\left(1+\sum_{i=1}^{L} g_{i}^{2} a_{i}^{2}\right)^{-1}\left(\sum_{i=1}^{L} g_{i} a_{i} \sqrt{\alpha_{i}}\right)^{2}$ a total SNR. Therefore, we can easily express the capacity as follows:

$$
\begin{aligned}
C=\frac{1}{2} \log \left(1+\left(1+\sum_{i=1}^{L} g_{i}^{2} a_{i}^{2}\right)^{-1}\right. \\
\left.\times\left(\sum_{i=1}^{L} g_{i} a_{i} \sqrt{\alpha_{i}}\right)^{2}\right) \\
=\frac{1}{2} \log (1+\beta) .
\end{aligned}
$$

Let $C\left(P_{1}, \ldots, P_{L}\right)$ denote the capacity achieved by optimally assigning $P_{i}$ to sensor $i$. Maximizing the capacity under a sum power constraint can be written as

$$
\begin{array}{ll}
\max _{P_{i} ; 1 \leq i \leq L} & C\left(P_{1}, \ldots, P_{L}\right) \\
\text { s.t. } & \sum_{i=1}^{L} a_{i}^{2}\left(\alpha_{i}^{-1}+1\right) \leq P, \quad 1 \leq i \leq L .
\end{array}
$$

Maximizing capacity in (18) is equivalent to maximizing the total SNR as follows:

$$
\begin{array}{ll}
\max _{a_{i}^{2} ; 1 \leq i \leq L} & \beta\left(a_{1}^{2}, \ldots, a_{L}^{2}\right) \\
\text { s.t. } & \sum_{i=1}^{L} a_{i}^{2}\left(\alpha_{i}^{-1}+1\right) \leq P, \quad 1 \leq i \leq L .
\end{array}
$$

With the aid of Appendix B that follows the solution in [24], we get the best achievable total SNR as

$$
\beta_{\mathrm{opt}}=\sum_{i=1}^{L} \frac{\alpha_{i}}{1+\left(\alpha_{i}^{-1}+1\right) /\left(g_{i}^{2} P\right)} .
$$

The optimal power allocation achieving the optimal total SNR is

$$
P_{i}^{\mathrm{opt}}=c_{i} P, \quad 1 \leq i \leq L,
$$

where

$$
\begin{gathered}
c_{i}=c \frac{g_{i}^{2} \alpha_{i}^{2}\left(\alpha_{i}^{-1}+1\right)}{\left(\alpha_{i}^{-1}+1+g_{i}^{2} P\right)^{2}}, \\
c=\left(\sum_{i=1}^{L} \frac{g_{i}^{2} \alpha_{i}^{2}\left(\alpha_{i}^{-1}+1\right)}{\left(\alpha_{i}^{-1}+1+g_{i}^{2} P\right)^{2}}\right)^{-1} .
\end{gathered}
$$

Implementing optimal power allocation, we need the FC to broadcast the constant $c$ and $P$ to the sensors. The sensors use $c, P$, and two local parameters, $g_{i}^{2}$ and $\alpha_{i}$, to determine their individual transmit power.

Therefore, we can express the optimal capacity of data collection as

$$
\begin{aligned}
C_{\mathrm{opt} 1} & =\frac{1}{2} \log \left(1+\beta_{\mathrm{opt}}\right) \\
& =\frac{1}{2} \log \left(1+\sum_{i=1}^{L} \frac{\alpha_{i}}{1+\left(\alpha_{i}^{-1}+1\right) /\left(g_{i}^{2} P\right)}\right) .
\end{aligned}
$$

\section{Capacity of Data Collection in Orthogonal MAC Model}

In this section, we adopt orthogonal channels between the sensors and the FC. We assume that the observed signal is analog and the observation noises are uncorrelated across sensors. In addition, we assume that the second moments of the signal and noise are known to the corresponding sensor and the FC. The FC deploys the MMSE estimator to generate estimates of the unknown signal. In this setting, we use an analog transmission system where observations are amplified and forwarded to the FC.

Suppose that the received signal of orthogonal MAC from sensor $i$ to FC can be written as

$$
y_{i}=g_{i} a_{i} s+g_{i} a_{i} n_{i}+v_{i}
$$

where $v_{i}$ and $g_{i}$ are the channel noise with zero mean and unit variance of channel $i$ and channel gain, respectively. For MMSE estimation, we can get an MSE, J, [24] as

$$
\frac{1}{J}=1+\sum_{i=1}^{L} \frac{g_{i}^{2} a_{i}^{2} \alpha_{i}}{1+g_{i}^{2} a_{i}^{2}}
$$

4.1. Capacity of Data Collection for Equal Power Allocation. For equal power method, $P_{i}=P / L$; thus we have $a_{i}^{2}=P_{i} /(1+$ $\left.\alpha_{i}^{-1}\right)$. By changing the form of (25), we get

$$
\frac{1}{J}=1+\sum_{i=1}^{L} \frac{\alpha_{i}}{1+\left(1+\alpha_{i}^{-1}\right) /\left(g_{i}^{2} P_{i}\right)} .
$$

Following the expression of (5) and $\sum_{i=1}^{L}\left(\alpha_{i} /(1+(1+\right.$ $\left.\left.\left.\alpha_{i}^{-1}\right) /\left(g_{i}^{2} P_{i}\right)\right)\right)$ as a total SNR of the system, we can write the capacity as

$$
C_{\text {eq2 }}=\frac{1}{2} \log \left(1+\sum_{i=1}^{L} \frac{\alpha_{i}}{1+\left(1+\alpha_{i}^{-1}\right) /\left(g_{i}^{2} P_{i}\right)}\right) .
$$


For $P_{i} \rightarrow \infty$, we can write an upper bound on the capacity of data collection in orthogonal MAC as

$$
\begin{aligned}
C_{\text {upp2 }} & =\frac{1}{2} \log \left(1+\sum_{i=1}^{L} \alpha_{i}\right) \\
& =\frac{1}{2} \log \left(1+\frac{L}{\sigma_{n}^{2}}\right) \\
& =C_{\text {upp } 1} .
\end{aligned}
$$

Interestingly, we can see that the upper bound on capacity of data collection for orthogonal MAC and that for the coherent MAC are equal.

4.2. Capacity of Data Collection for Optimal Power Allocation. To maximize the capacity of data collection on orthogonal MAC under optimal power method, first, we need to minimize the MSE under total power constraint, $P$. The MSE for optimal power method of the orthogonal MAC for the case of scalar source and observations is given in [24]

$$
\frac{1}{J}=1+\sum_{i=1}^{L_{1}} \alpha_{i}\left(1-\frac{\sqrt{1+\alpha_{i}^{-1}}}{\mathcal{c}_{0} g_{i}}\right)
$$

where $c_{0}$ and $L_{1}$ are the threshold of $g_{i}^{2} /\left(1+\alpha_{i}^{-1}\right) \geq 1 / c_{0}^{2}$ whether a sensor transmits or keeps silent and the number of active sensors, respectively. The threshold $c_{0}$ is defined by

$$
c_{0}=\frac{\left(\sum_{m=1}^{L_{1}}\left(\alpha_{m}\left(\alpha_{m}^{-1}+1\right) / g_{m}^{2}\right)+P\right)}{\left(\sum_{m=1}^{L_{1}}\left(\alpha_{m} \sqrt{\alpha_{m}^{-1}+1} / \sqrt{g_{m}^{2}}\right)\right)} .
$$

Following (5), we can express the capacity as,

$$
C_{\mathrm{opt} 2}=\frac{1}{2} \log \left(1+\sum_{i=1}^{L_{1}} \alpha_{i}\left(1-\frac{\sqrt{1+\alpha_{i}^{-1}}}{c_{0} g_{i}}\right)\right) .
$$

We note that the optimal power method for orthogonal MAC will allocate most of power to sensors that have good observation and channel qualities. Hence, the active sensors are sensors that have good observation and channel qualities.

\section{Simulation Results}

In Figure 2, we plot the curves of capacity of data collection for coherent MAC model versus total transmit power $P$ in $\mathrm{dB}$ (relative to the channel noise power) with the number of sensors $L=10$. In the simulation, sensor observation noise variance is set as $\sigma_{n}^{2}=0.5$. The channel gains, $g_{i}$, are taken as $c_{g} * d^{-\epsilon}$ where $d$ is uniformly taken from real interval $[1,10]$ and $\epsilon$ is a path loss parameter that we assume $\epsilon=2$. Parameter $c_{g}$ is a normalization constant to make $E\left(g_{i}\right)=$ 1. Simulations are averaged over 5000 realizations. Those parameters are also used in all simulations. For coherent MAC model in Figure 2, we can see that when $P$ increases,

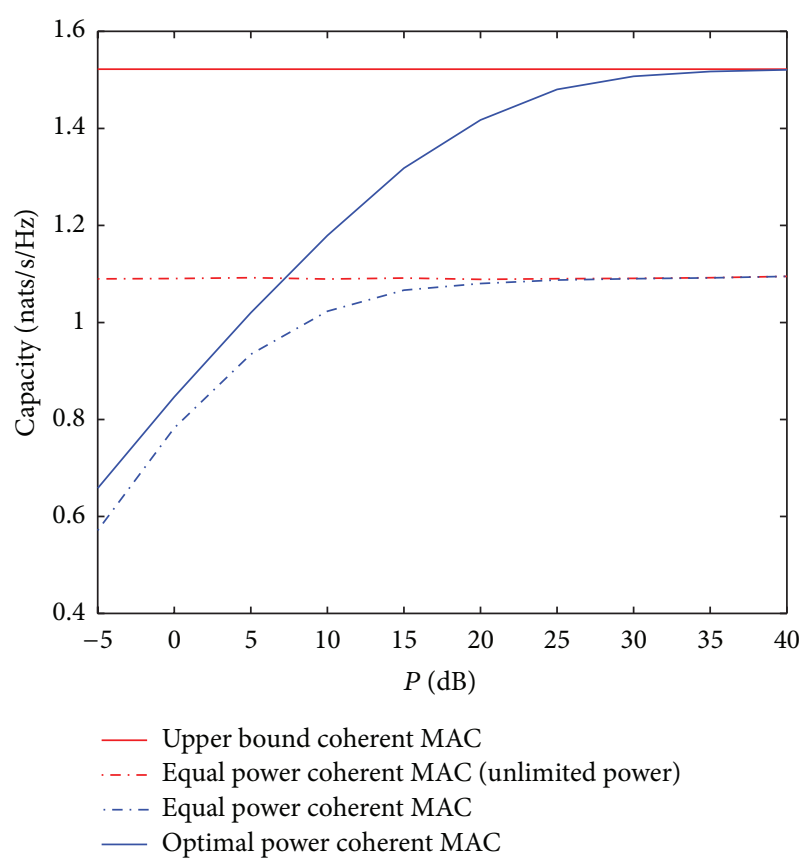

FIGURE 2: Capacity of data collection for equal power method versus optimal power method in coherent MAC as $P$ increases. Note that power $P$ is taken relative to the channel noise power. Since we assume that the channel noise has unitary variance, thus we label the total transmit power in unit of $\mathrm{dB}$.

equal power method and optimal power method converge to two different limits that are $C_{\text {eq1 }}(\infty)$ and $C_{\text {upp } 1}$, respectively. This is because the optimal power method allocates power by taking into account channel gain sensor observation while the equal power method does not. Moreover, the limit of the equal power method, $C_{\text {eql }}(\infty)$, is due to inhomogeneous sensing environment.

We can see in Figure 3 for orthogonal MAC model that the capacity of the optimal power method is larger than that of the equal power method. This is because the optimal power method allocates most of power to sensors that have good observation and channel qualities. Moreover, as $P$ increases, both the optimal method and the equal one converge to the upper bound. In high power regime, each sensor has a redundant power to transmit the sensing data and can easily combat the channel noise.

In Figure 4, we compare the optimal power method for both MAC models. We can see that the optimal power method for coherent MAC outperforms the orthogonal MAC. This is a consequence of using orthogonal links that have $L$ different channel noises. We also compare the equal method of both MAC in Figure 5. In high power regime $(P>$ $15 \mathrm{~dB}$ ), the equal method for orthogonal has larger capacity because the coherent MAC is limited by the finite number of sensor observations.

We simulate the capacity versus the number of sensors $L$ with the total power being constant at $P=20 \mathrm{~dB}$ (relative to channel noise variance) for both models in Figure 6. The capacity of both models increases as the total number of 


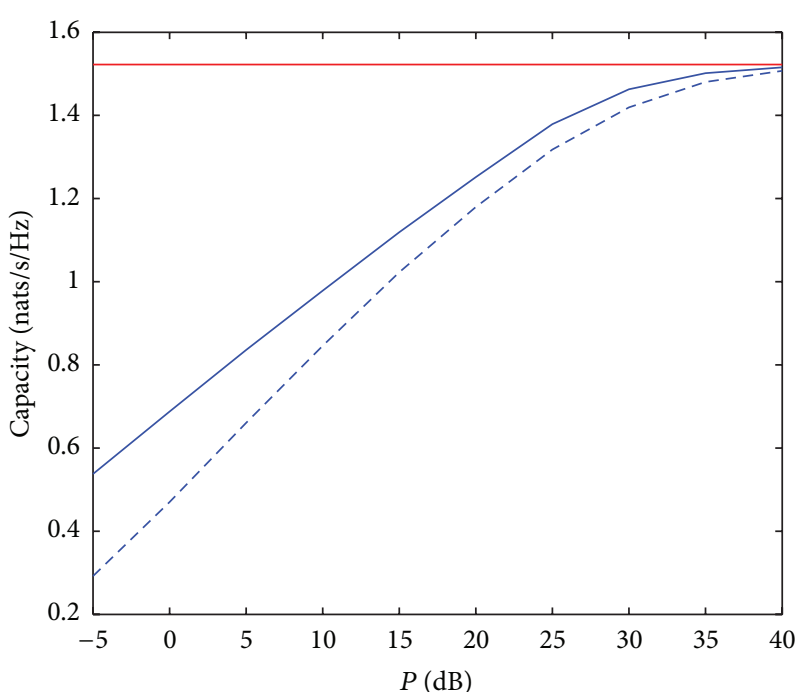

- Upper bound orthogonal MAC
- - Equal power orthogonal MAC
- Optimal power orthogonal MAC

FIGURE 3: Capacity of data collection for equal power method versus optimal power method in orthogonal MAC as $P$ increases. Note that power $P$ is taken relative to the channel noise power. Since we assume that the channel noise has unitary variance, thus we label the total transmit power in unit of $\mathrm{dB}$.

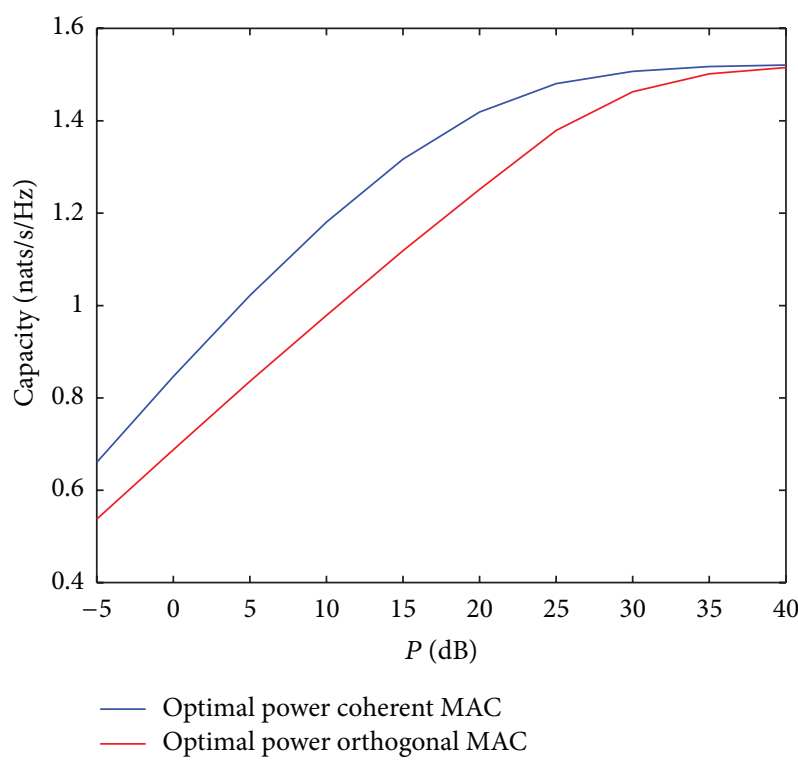

FIGURE 4: Comparison between the capacity of data collection in coherent MAC and orthogonal MAC for optimal power allocation method.

sensors increases. This is because as the number of sensors increases the total SNR also increases. However, we can see that, with this finite total power and a large number of sensors, the capacity of the coherent MAC is larger than that of the orthogonal MAC for both methods, equal and optimal power. This is because the corrupted channels in orthogonal

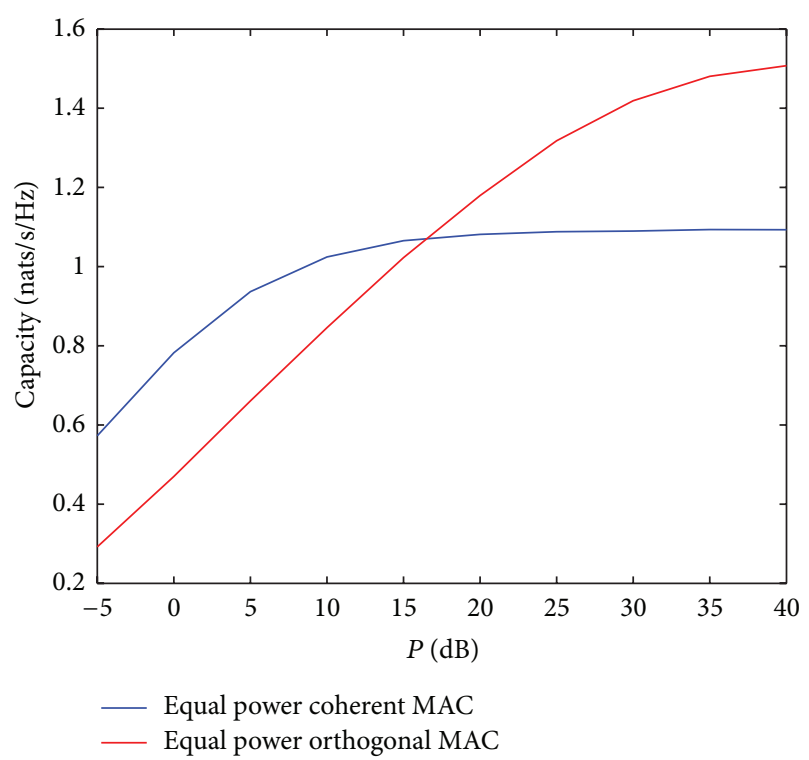

FIGURE 5: Comparison between the capacity of data collection in coherent MAC and orthogonal MAC for equal power allocation method.

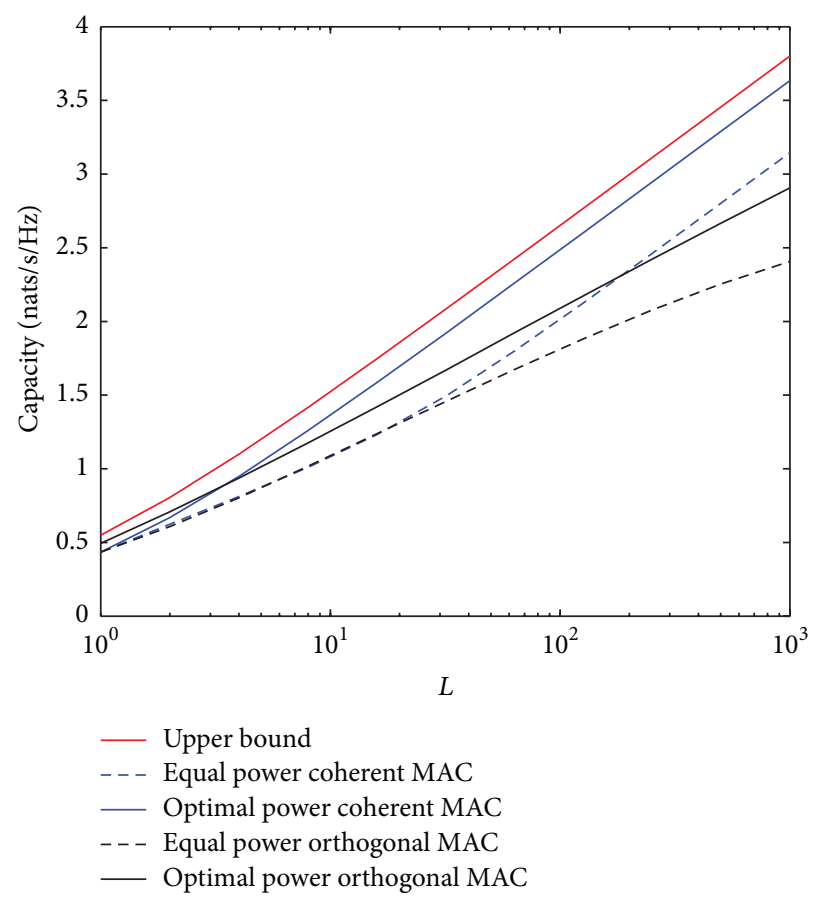

Figure 6: Capacity of data collection of equal power method versus optimal power method as $L$ increases for either coherent and orthogonal MAC in a finite power budget, $P=20 \mathrm{~dB}$.

MAC cannot be eliminated even when $L$ goes to infinity. However, in the corehent MAC model, channel noise incurs only once per reception at FC.

In Figure 7, we plot the percentage of active sensors versus the total transmission power, where we set $L=100$ in the simulation for optimal method in orthogonal MAC. We note that the number of active sensors is less than $L$ 


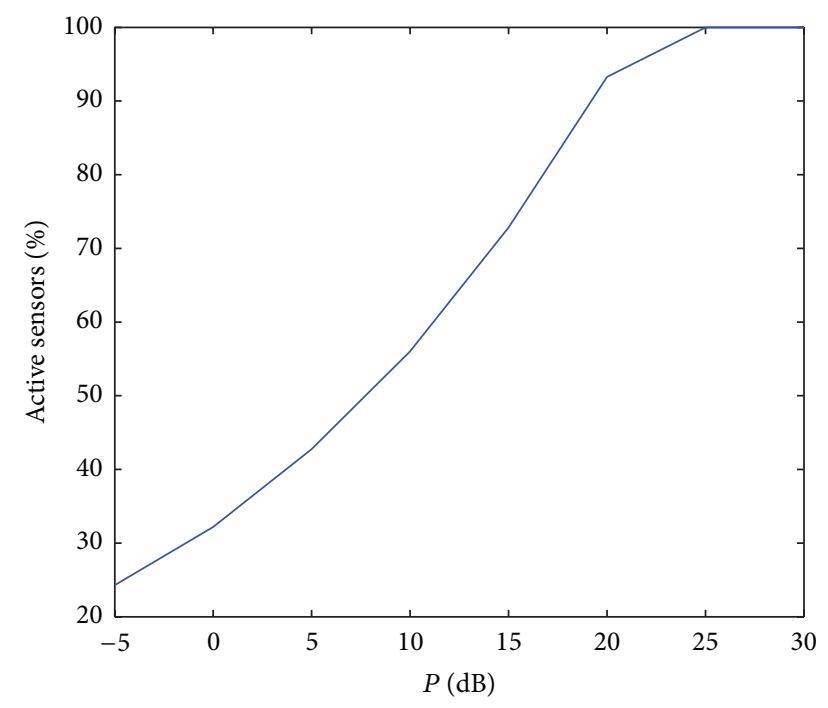

FIgURe 7: Percentage of active sensors as $P$ increases for optimal power method in orthogonal MAC.

when the total power budget is small. This confirms that the optimal power allocation for orthogonal MAC allocates most of power to only the sensors that have good observation and channel qualities. Activating only the sensors that have good observation and channel qualities can be used to conserve energy of the sensors and extend sensor's lifetime.

\section{Conclusion}

We studied the capacity of data collection in wireless sensor networks by considering power allocation strategy. We considered a scenario in which a number of sensors observe a target being estimated at fusion center (FC) using minimum mean-square error (MMSE) estimator. Based on the relationship between mutual information and minimum mean-square error (I-MMSE), we derived the capacity of data collection in both coherent MAC model and orthogonal MAC model. Considering power constraint, we derived the capacity under two scenarios: equal power allocation and optimal power allocation of both models. We also provided the upper bound of capacity as a benchmark. In particular, we showed that the capacity of data collection scaled as $\Theta((1 / 2) \log (1+L))$ when the number of sensors $L$ grows to infinity. We verified the capacity calculation by simulation results as follows. (1) For coherent MAC model, we derived the optimal power allocation strategy that maximizes the capacity. The capacity of the optimal power is larger than that of the equal power because the optimal power method takes into account the SNR observation and channel gain to determine their individual transmit power. (2) For orthogonal MAC model, we derived the optimal power allocation strategy that maximizes the capacity. The capacity of the optimal power is larger than that of the equal power because the optimal power method allocates most of power to only the sensors that have good SNR observation and channel qualities, while the sensors with bad observation and bad channel qualities will be turned off. Turning off the sensors with bad observation and bad channel qualities can be used to conserve energy of the sensors and extend sensor's lifetime. Moreover, we showed that the capacity of coherent MAC is larger than that of orthogonal MAC, particularly when the number of sensors $L$ is large and the total power $P$ is fixed. This is consequence of using orthogonal link from the sensors to FC where the corrupted channel cannot be eliminated even when $L$ goes to infinity.

\section{Appendices}

\section{A. Derivation of the Lower Bound of MMSE}

Instead of intuitive assumption, we provide analytical derivation of the lower bound on MMSE estimation that can be achieved when the total power $P \rightarrow \infty$ and the channel gain $g_{i}=1$. As $g_{i}=1$, we can write (9) as

$$
\frac{1}{J}=1+\left(1+\sum_{i=1}^{L} a_{i}^{2}\right)^{-1}\left(\sum_{i=1}^{L} a_{i} \sqrt{\alpha_{i}}\right)^{2} .
$$

Based on Cauchy-Schwarz inequality [27] that $\left(\sum_{i=1}^{n} k_{i} l_{i}\right)^{2} \leq$ $\left(\sum_{i=1}^{n} k_{i}^{2}\right)\left(\sum_{i=1}^{n} l_{i}^{2}\right)$, we can rewrite (A.1) as

$$
\frac{1}{J} \leq 1+\left(1+\sum_{i=1}^{L} a_{i}^{2}\right)^{-1}\left(\sum_{i=1}^{L} a_{i}^{2}\right)\left(\sum_{i=1}^{L} \alpha_{i}\right) .
$$

We have power amplification factor, $a_{i}=\sqrt{P / L\left(\alpha_{i}^{-1}+1\right)}$; then we have

$$
\begin{aligned}
\frac{1}{J} \leq & +\left(1+P \sum_{i=1}^{L} \frac{1}{L\left(\alpha_{i}^{-1}+1\right)}\right)^{-1} \\
& \times\left(P \sum_{i=1}^{L} \frac{1}{L\left(\alpha_{i}^{-1}+1\right)}\right)\left(\sum_{i=1}^{L} \alpha_{i}\right) .
\end{aligned}
$$

Thus, as $P \rightarrow \infty,\left(P \sum_{i=1}^{L}\left(1 /\left(L\left(\alpha_{i}^{-1}+1\right)\right)\right)\right) /\left(1+P \sum_{i=1}^{L}\right.$ $\left.\left(1 /\left(L\left(\alpha_{i}^{-1}+1\right)\right)\right)\right) \rightarrow 1$. Then, the lower bound of MMSE is

$$
\frac{1}{J_{0}}=1+\sum_{i=1}^{L} \alpha_{i}
$$

\section{B. Derivation of the Optimal SNR}

We know that (19) is not a convex problem, but we can transform it into an equivalent convex form. Let us suppose the optimal solution of $(19)$ is $\beta_{\text {opt }}(P)$; then $1 / \beta_{\text {opt }}(P)$ should be monotonically decreasing as $P$ increases. Then, we reform the objective function in (19) as minimizing the total power consumption subject to a given inverse of total SNR constraint as follows:

$$
\begin{array}{ll}
\min _{P_{i}} & P=\sum_{i=1}^{L} P_{i} \\
\text { s.t. } & \frac{1}{\beta\left(P_{1}, \ldots, P_{L}\right)} \leq \frac{1}{\beta} .
\end{array}
$$


In terms of power amplification factor $a_{i}$ and straightforward modification of (B.1), we get

$$
\begin{array}{ll}
\min _{a_{i}, \ldots, a_{L}} & \sum_{i=1}^{L} a_{i}^{2}\left(1+\alpha_{i}^{-1}\right) \\
\text { s.t. } & \left(1+\sum_{i=1}^{L} g_{i}^{2} a_{i}^{2}\right)^{-1}\left(\sum_{i=1}^{L} g_{i} a_{i} \sqrt{\alpha_{i}}\right)^{2} \geq \beta^{-1} .
\end{array}
$$

We know that (B.2) is still convex in terms of $a_{i i=1}^{L}$. Therefore, we use a slack variable $t=\sum_{i=1}^{L} g_{i} a_{i} \sqrt{\alpha_{i}}$ as

$$
\begin{array}{ll}
\min _{a_{i}, \ldots, a_{L}} & \sum_{i=1}^{L} a_{i}^{2}\left(1+\alpha_{i}^{-1}\right) \\
\text { s.t. } & \left(1+\sum_{i=1}^{L} g_{i}^{2} a_{i}^{2}\right) \geq \beta t^{2} \\
& \sum_{i=1}^{L} g_{i} a_{i} \sqrt{\alpha_{i}}-t=0 .
\end{array}
$$

The Lagrangian function for (B.3) is

$$
\begin{aligned}
\mathscr{L}\left(a_{i}, t, \mu, \eta\right)= & \sum_{i=1}^{L} a_{i}^{2}\left(1+\alpha_{i}^{-1}\right) \\
& +\mu\left(1+\sum_{i=1}^{L} g_{i}^{2} a_{i}^{2}-\beta t^{2}\right) \\
& +\eta\left(t-\sum_{i=1}^{L} g_{i} a_{i} \sqrt{\alpha_{i}}\right),
\end{aligned}
$$

where $\eta \in \mathbb{R}$ and $\mu \geq 0$. From the Lagrangian function, we can derive solution based on KTT condition [28]:

$$
\begin{gathered}
\frac{\partial \mathscr{L}}{\partial t}=-2 \mu \beta t+\eta=0 \\
\frac{\partial \mathscr{L}}{\partial a_{i}}=2\left(1+\alpha_{i}^{-1}+\mu g_{i}^{2}\right) a_{i}-g_{i} \sqrt{\alpha_{i}} \eta=0, \quad 1 \leq i \leq L, \\
\mu\left(1+\sum_{i=1}^{L} g_{i}^{2} a_{i}^{2}-\beta t^{2}\right)=0 \\
\sum_{i=1}^{L} g_{i} a_{i} \sqrt{\alpha_{i}}=0 .
\end{gathered}
$$

From the second KKT conditions; we have

$$
a_{i}=\frac{g_{i} \sqrt{\alpha_{i}} \eta}{2\left(1+\alpha_{i}^{-1}+\mu g_{i}^{2}\right)} \text {. }
$$

We use the slack variable $t=\sum_{i=1}^{L} g_{i} a_{i} \sqrt{\alpha_{i}}$ and (B.6) into the first KTT conditions, we get

$$
\sum_{i=1}^{L} \frac{\mu g_{i}^{2} \alpha_{i}}{1+\alpha_{i}^{-1}+\mu g_{i}^{2}}=\beta^{-1} .
$$

Now, we plug in the first KKT conditions, $t=\left(\left(\beta^{-1}\right) \eta\right) / 2 \mu$, and (B.6) into the third KTT conditions as

$$
\eta=2\left(\frac{\beta^{-1}}{\mu^{2}}-\sum_{i=1}^{L} \frac{g_{i}^{4} \alpha_{i}}{\left(1+\alpha_{i}^{-1}+\mu g_{i}^{2}\right)^{2}}\right)^{-1 / 2} .
$$

Thus, the optimal power allocation of sensor $i$ in terms of $\mu$ and $\eta$ is

$$
P_{i}=a_{i}^{2}\left(1+\alpha_{i}^{-1}\right)=\frac{\eta^{2}}{4} \frac{g_{i}^{2} \alpha_{i}\left(1+\alpha_{i}^{-1}\right)}{\left(1+\alpha_{i}^{-1}+\mu g_{i}^{2}\right)^{2}},
$$

where we have

$$
\begin{aligned}
P & =\sum_{i=1}^{L} P_{i} \\
& =\frac{\eta^{2}}{4} \underbrace{\sum_{i=1}^{L} \frac{g_{i}^{2} \alpha_{i}\left(1+\alpha_{i}^{-1}\right)}{\left(1+\alpha_{i}^{-1}+\mu g_{i}^{2}\right)^{2}}}_{=c^{-1}}
\end{aligned}
$$

$$
\stackrel{(a)}{=} \mu
$$

By direct calculation that involves (B.7), (B.8), and (B.9), (a) is held. From (B.6), we obtain that the optimal SNR, $\beta_{\text {opt }}$, is a function of the total power, $P$, as

$$
\beta_{\mathrm{opt}}=\sum_{i=1}^{L} \frac{\alpha_{i}}{1+\left(\alpha_{i}^{-1}+1\right) /\left(g_{i}^{2} P\right)} .
$$

\section{Conflict of Interests}

The authors declare that there is no conflict of interests regarding the publication of this paper.

\section{References}

[1] C. Wang, C. Jiang, Y. Liu, X. Li, and S. Tang, "Aggregation capacity of wireless sensor networks: extended network case," IEEE Transactions on Computers, no. 99, pp. 1-10, 2012.

[2] S. Chen, M. Huang, S. Tang, and Y. Wang, "Capacity of data collection in arbitrary wireless sensor networks," IEEE Transactions on Parallel and Distributed Systems, vol. 23, no. 1, pp. 5260, 2012.

[3] P. Santi, "On the data gathering capacity and latency in wireless sensor networks," IEEE Journal on Selected Areas in Communications, vol. 28, no. 7, pp. 1211-1221, 2010.

[4] D. Marco, E. J. Duarte-Melo, M. Liu, and D. L. Neuhoff, "On the many-to-one transport capacity of a dense wireless sensor network and the compressibility of its data," Lecture Notes in Computer Science, vol. 2634, pp. 1-16, 2003.

[5] E. J. Duarte-Melo and M. Liu, "Data-gathering wireless sensor networks: organization and capacity," Computer Networks, vol. 43, no. 4, pp. 519-537, 2003.

[6] H. El Gamal, "On the scaling laws of dense wireless sensor networks: the data gathering channel," IEEE Transactions on Information Theory, vol. 51, no. 3, pp. 1229-1234, 2005. 
[7] K. Zheng and R. Barton, "Toward optimal data aggregation in random wireless sensor networks," in Proceedings of the 26th IEEE International Conference on Computer Communications (IEEE INFOCOM '07), pp. 249-257, May 2007.

[8] B. Liu, D. Towsley, and A. Swami, "Data gathering capacity of large scale multihop wireless networks," in Proceedings of the 5th IEEE International Conference on Mobile Ad-Hoc and Sensor Systems (MASS '08), pp. 124-132, October 2008.

[9] S. Chen, Y. Wang, X.-Y. Li, and X. Shi, "Capacity of data collection in randomly-deployed wireless sensor networks," Wireless Networks, vol. 17, no. 2, pp. 305-318, 2011.

[10] H. Zheng, S. Xiao, X. Wang, X. Tian, and M. Guizani, "Capacity and delay analysis for data gathering with compressive sensing in wireless sensor networks," IEEE Transactions on Wireless Communications, vol. 12, no. 2, pp. 917-927, 2013.

[11] P. Gupta and P. R. Kumar, "The capacity of wireless networks," IEEE Transactions on Information Theory, vol. 46, no. 2, pp. 388404, 2000.

[12] S. Marano, V. Matta, and P. Willett, "Distributed estimation in large wireless sensor networks via a locally optimum approach," IEEE Transactions on Signal Processing, vol. 56, no. 2, pp. 748756, 2008.

[13] A. Ribeiro and G. B. Giannakis, "Bandwidth-constrained distributed estimation for wireless sensor networks-part I: gaussian case," IEEE Transactions on Signal Processing, vol. 54, no. 3, pp. 1131-1143, 2006.

[14] A. Ribeiro and G. B. Giannakis, "Bandwidth-constrained distributed estimation for wireless sensor networks-part II: unknown probability density function," IEEE Transactions on Signal Processing, vol. 54, no. 7, pp. 2784-2796, 2006.

[15] Z.-Q. Luo, "Universal decentralized estimation in a bandwidth constrained sensor network," IEEE Transactions on Information Theory, vol. 51, no. 6, pp. 2210-2219, 2005.

[16] J.-J. Xiao and Z.-Q. Luo, "Decentralized estimation in an inhomogeneous sensing environment," IEEE Transactions on Information Theory, vol. 51, no. 10, pp. 3564-3575, 2005.

[17] K. Liu, H. E. Gamal, and A. M. Sayeed, "On optimal parametric field estimation in sensor networks," in Proceedings of the IEEE/ SP 13th Workshop on Statistical Signal Processing, pp. 1170-1175, July 2005.

[18] G. Mergen and L. Tong, "Type based estimation over multiaccess channels," IEEE Transactions on Signal Processing, vol. 54, no. 2, pp. 613-626, 2006.

[19] M. K. Banavar, C. Tepedelenlioğlu, and A. Spanias, "Estimation over fading channels with limited feedback using distributed sensing," IEEE Transactions on Signal Processing, vol. 58, no. 1, pp. 414-425, 2010.

[20] H. Şenol and C. Tepedelenlioğlu, "Performance of distributed estimation over unknown parallel fading channels," IEEE Transactions on Signal Processing, vol. 56, no. 12, pp. 6057-6068, 2008.

[21] T. J. Goblick, "Theoretical limitations on the transmission of data from analog sources," IEEE Transactions on Information Theory, vol. 11, no. 4, pp. 558-567, 1965.

[22] M. Gasfpar, B. Rimoldi, and M. Vetterli, "To code, or not to code: lossy source-channel communication revisited," IEEE Transactions on Information Theory, vol. 49, no. 5, pp. 1147-1158, 2003.

[23] M. Gastpar, "Uncoded transmission is exactly optimal for a simple Gaussian "sensor" network," IEEE Transactions on Information Theory, vol. 54, no. 11, pp. 5247-5251, 2008.
[24] J.-J. Xiao, S. Cui, Z.-Q. Luo, and A. J. Goldsmith, "Linear coherent decentralized estimation," IEEE Transactions on Signal Processing, vol. 56, no. 2, pp. 757-770, 2008.

[25] S. Cui, J.-J. Xiao, A. J. Goldsmith, Z.-Q. Luo, and H. V. Poor, "Estimation diversity and energy efficiency in distributed sensing," IEEE Transactions on Signal Processing, vol. 55, no. 9, pp. 4683-4695, 2007.

[26] D. Guo, S. Shamai, and S. Verdú, "Mutual information and minimum mean-square error in Gaussian channels," IEEE Transactions on Information Theory, vol. 51, no. 4, pp. 1261-1282, 2005.

[27] J. M. Steele, The Cauchy-Schwarz Master Class, Cambridge University Press, Cambridge, Mass, USA, 2004.

[28] S. Byod and L. Vandenberghe, Convex Optimization, Cambridge University Press, Cambridge, Mass, USA, 2003. 

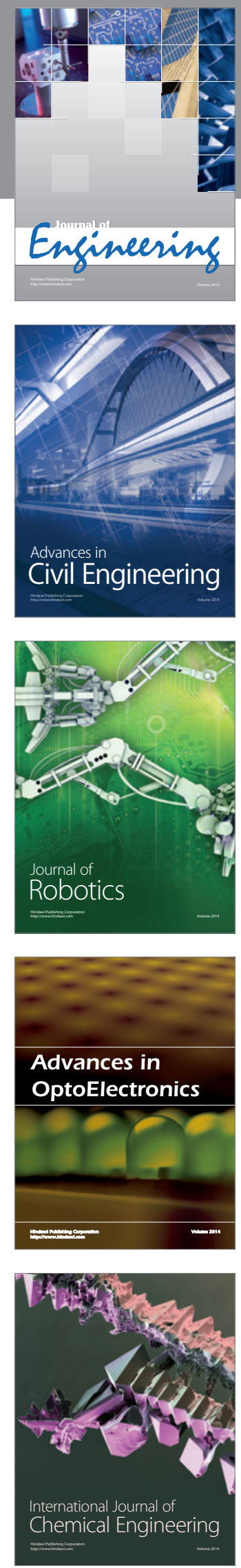

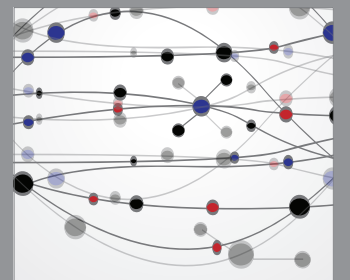

The Scientific World Journal
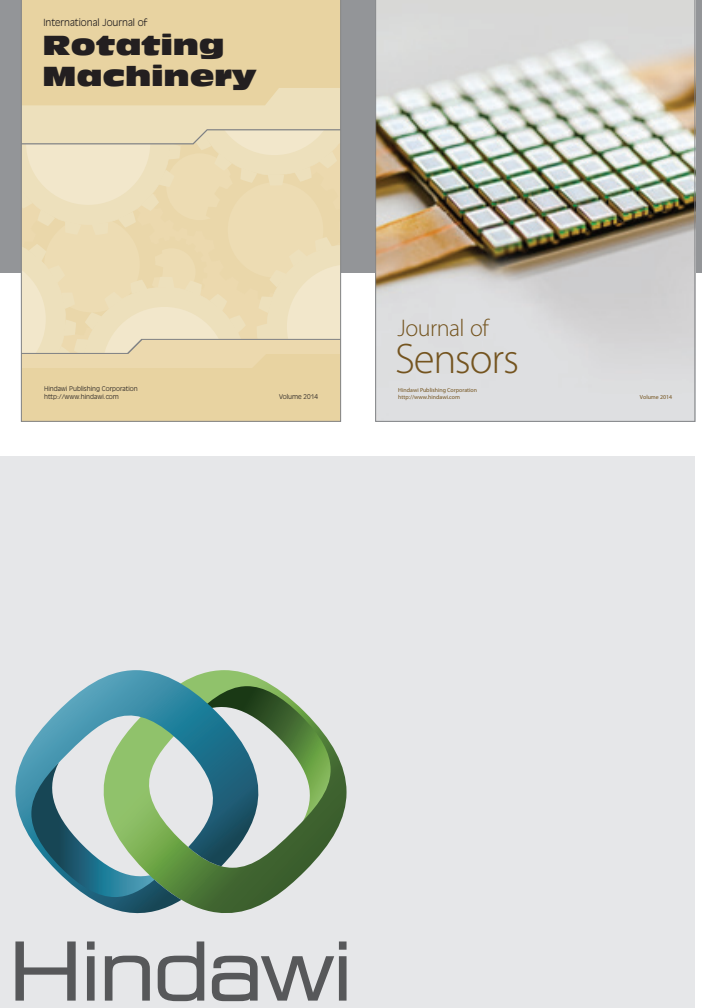

Submit your manuscripts at http://www.hindawi.com
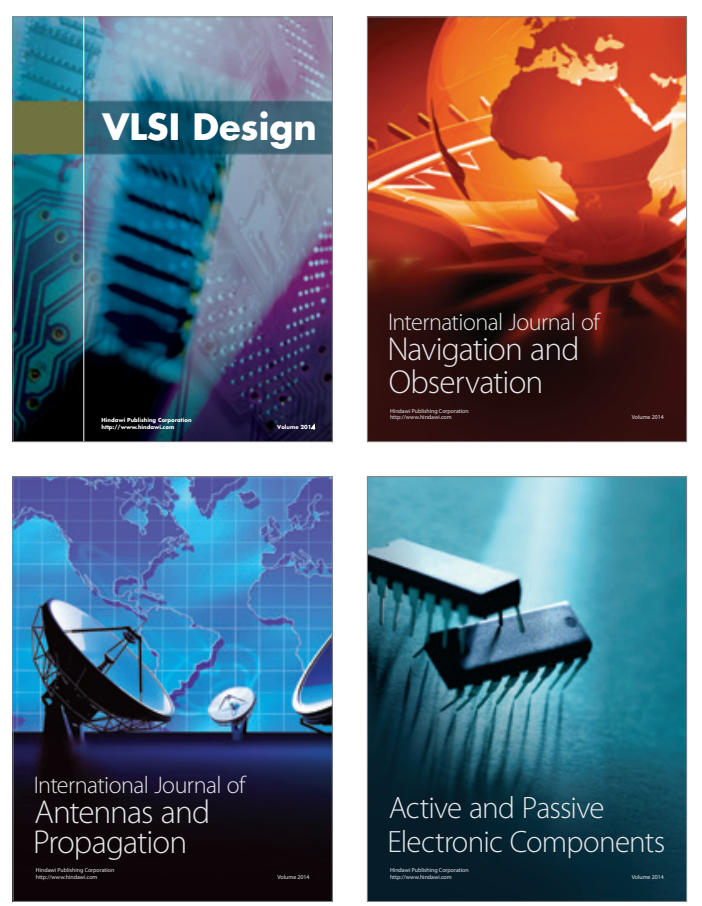
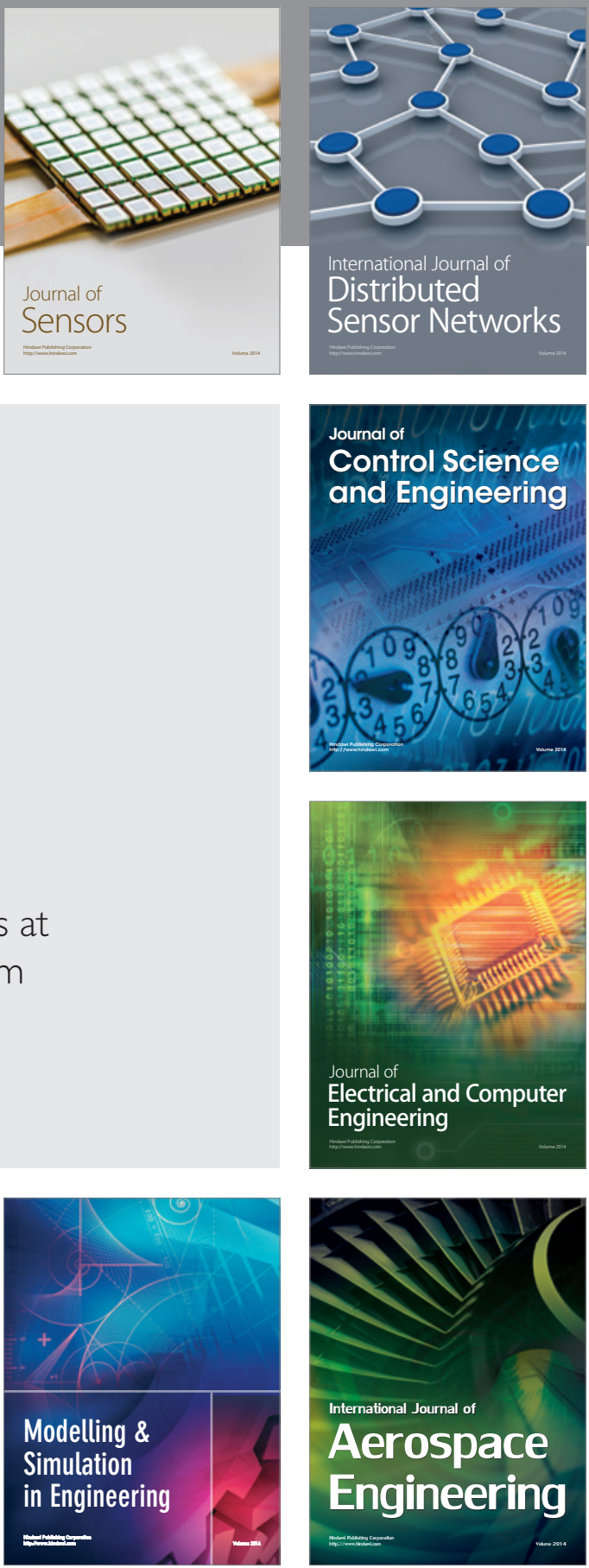

Journal of

Control Science

and Engineering
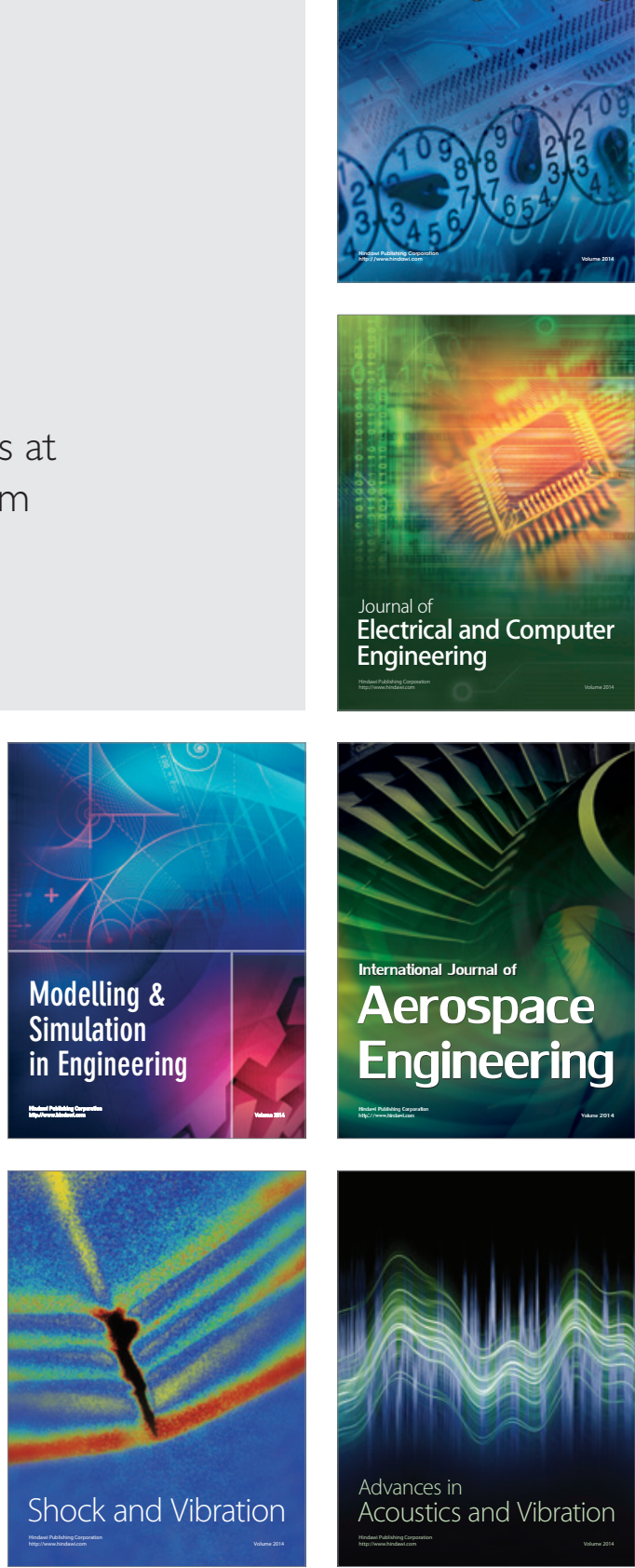